\title{
EFFECT OF DISPLACED GEOMAGNETIC AND GEOGRAPHIC POLES ON HIGH-LATITUDE PLASMA CONVECTION AND IONOSPHERIC DEPLETIONS
}

\section{J. J. Sojka, W. J. Raitt, and R. W. Schunk}

Center for Atmospheric and Space Sciences, Utah State University, Logan, Utah 84322

Abstract. We assumed that the ionospheric $\frac{\text { Abstract }}{\text { at }}$ high latitudes has a tendency to plasma at about the geographic pole and that corotate abic convection is relative to the agnetosphetic pole. With this assumption we geomagne plasma drift patterns over the polar calculated a range of constant magnetospheric cap for a ranger as well as for asymmetric electric fields with enhanced plasma flow on elther the dawnside or the duskside of the polar efther calculated the drift patterns in both the geographic inertial and the geomagnetic inertial frame taking into account the displacement between the geographic and geomagnetic poles. We found that this displacement between the poles has an important effect on the plasma drift patterns. In particular, we found the following: (1) A timeindependent magnetospheric electric field produces a flow pattern in the magnetic inertial frame that does not vary with universal time. (2) This flow pattern becomes UT dependent in the geographic inertial frame because of the sotion of the geomagnetic pole about the geographic pole. (3) The UT variation of the plasma flow pattern in the geographic inertial frame occurs on a time scale that is comparable to satellite orbital periods and that is much less than typical plasma convection flow times over the polar cap. (4) In the geographic inertial frame the main region of very low speed flow is not centered at 1800 LT but moves from about 1300 to $2300 \mathrm{LT}$ during the course of a day. (5) In the geographic inertial frame a throatlike feature appears at certain universal times owing to the relative motion of the geographic and geomagnetic poles. This feature is not seen in the geomagnetic inertial frame and is not connected with our model of the aagnetospheric electric field. These results and others described in the paper have important inplications for both the interpretation of satellite data related to high-latitude lonospheric dynamics and the formation of ionospheric troughs.

\section{Introduction}

One of the most distinctive features of the nighttime high-latitude ionosphere is the do-called 'mid-latitude' or 'main' electron This trough [Muldrew, 1965; Liszka, 1965]. very trough corresponds to a narrow region of equatow electron density situated just is generard of the nocturnal auroral oval. It and less less evident in summer and spring.

add-latice the initial discovery of the theoretitude trough, there have been numerous Cxperimental studies dealing

\footnotetext{
Paper Ight 1979 by the American Geophysical Union.

with both the morphology and the formation of the trough [Sharp, 1966; Miller, 1970; Rycroft and Thomas, 1970; Tulunay and Sayers, 1971; Feinblum and Horan, 1973; Grebowsky et a.l., 1974; Knudsen, 1974; Banks et al., 1974; Wrenn and Raitt, 1975; Schunk and Banks, 1975; Schunk et a1., 1975, 1976; Knudsen et a1, 1977; Wagner et a1., 1977; Spiro et al., 1978; Watkins, 1978; Brinton et al., 1978; Ahmed et al., 1979]. These studies indicate that the trough is a rather complex feature which displays considerable temporal and spatial variation. For example, the observations of Feinblum and Horan [1968] indicate that the trough is deepest near midnight, those of Wagner et a1. [1973] indicate a minimum in trough density in the early morning, while the recent Atmosphere Explorer measurements of Brinton et al. [1978] show the trough to be deepest near dusk.

The temporal and spatial variation of the trough undoubtedly results from the competition between a variety of processes known to be operating within the trough region [cf. Schunk et al., 1976]. In the absence of sunlight the ionosphere decays owing to ordinary ionic recombination. However, vertical drifts due to either neutral winds or electric fields will act to deplete or maintain the ionosphere depending on whether the induced drift is downward or upward. Solar radiation resonantly scattered into the nightside hemisphere will act to maintain trough densities, while polar wind escape, enhanced $\mathrm{N}_{2}$ densities, and increased ion temperatures will ${ }^{2}$ act to deplete the trough. Also, vibrating $\mathrm{N}_{2}$ molecules created in the nocturnal auroral oval and transported equatorward by diffusion and the thermospheric wind will act to reduce trough densities.

Although numerous processes may act within the trough region, the formation of the trough is no doubt intimately linked with the plasma convection pattern. In an initial study, Knudsen [1974] suggested that the trough results from ordinary ionic recombination coupled with long decay times. In Knudsen's study the plasma convection paths in the high-latitude ionosphere were determined by the competition between corotation and a two-cell convection pattern due to the solar wind-magnetosphere interaction [Axford and Hines, 1961]. In Knudsen's mode1 this competition produced a stagnation point at trough latitudes at 1800 MLT. The long decay times for trough plasma occurred because the plasma convected across the polar cap and nocturnal auroral oval, turned westward near midnight, stagnated near 1800 MLT, and then essentially corotated around the nightside. In the winter hemisphere most of this convection path is in darkness.

In a subsequent investigation, Schunk et al. [1976] studied the extent to which the various processes described above could account for the 
observed troughs. These authors found that in combination the various destructive processes could readily account for the great variety of troughs found by experimentation. However, they also found that the very deep ionization troughs that are frequently observed near midnight could be explained only by the lack of photo-ionization coupled with ionic recombination and slow convection.

In a more recent study, Spiro et al. [1978] accepted Knudsen's trough formation mechanism but suggested a different time history for the plasma in the premidnight trough. These authors proposed that the plasma in the trough corotated eastward into the evening local time sector from dusk before stagnating and flowing westward at higher latitudes in the dusk-midnight sector. The proposed drift pattern can be obtained from the competition between corotation and magnetospheric convection if the two-ce 11 convection pattern is tilted with respect to the noon-midnight meridian in such a way as to move the stagnation point from 1800 MLT toward midnight.

Watkins [1978] also accepted Knudsen's basic trough formation mechanism, and using a similar convection pattern, he calculated contours of electron density at $300 \mathrm{~km}$ over the polar region for low sunspot number and quiet geomagnetic conditions, i.e., maximum electric field strengths of $20-30 \mathrm{mV} \mathrm{m}$. Watkins allowed for the displacement between the geomagnetic and geographic poles and was able to show that the resultant universal time (UT) response of the ionosphere produces large diurnal changes in both the polar cap densities and trough morphology. He also studied the seasonal behavior of the polar region and the response of the polar cap ionization to asymmetries in the magnetospheric electric field pattern.

The purpose of this brief report is to show that the displacement between the geographic and geomagnetic poles affects significantly the competiton between corotation and magnetospheric convection in a way not fully appreciated by Watkins [1978] and that this in turn has important implications not only for the formation of the premidnight ionization trough but for ionospheric depletions in general.

Plasma Drift Trajectories and Speed Distribution

It is evident from the previous section that a study of ionization in the polar regions requires a knowledge of the plasma drift trajectories and speed distribution, which are determined by the combined action of the magnetospheric electric field and corotation. However, the magnetospheric electric field mapping into the ionosphere is determined by the geomagnetic axis, while corotation is relative to the geographic axis. Since these two axes are not aligned but subtend an angle of about $11.5^{\circ}$ in the northern hemisphere, the eventual plasma drift velocity may well depend on the relative location of the axes.

To study the effect of displaced geomagnetic and geographic axes on the plasma drift velocity, it was sufficient to consider a simple corotation-magnetospheric convection model. In this model we assumed that the ionospheric plasma tends to corotate about the geographic
axis. A rigorous justification assumption is difficult; however, the of this ionosphere corotates owing to strong coupling the neutral atmosphere, while at lating to equatorward of the auroral zones, where convection is small, corotation is obs the throughout the $\mathrm{E}$ and $\mathrm{F}$ regions. The tendencyed the plasma to corotate competes of magnetospheric convection, and for the with process we used a simplified form of Volland model of the magnetospheric electric [Volland, 1975, 1978]. Within the polar enclosed by the $77^{\circ}$ magnetic latitude contour the electric field is constant along the dawn-dusk meridian. The total potential dhe which has a maximum value at $77^{\circ}$ latitude on the dawn magnetic meridian and a minimum value at $77^{\circ}$ latitude on the dusk magnetic meridian, is the only free parameter in the model. Outside the polar region the field falls off as the inverse fourth power of sine co-latitude. Differing degrees of magnetic activity are selected by changing the total potential drop in the magnetospheric convection model. The effect of asymmetric convection models will be discussed later.

To combine magnetospheric convection and corotation, it was convenient to first transform the corotation velocity from the geographic inertial frame to the geomagnetic inertial frame and then add it to the $\underline{E} \times \underline{B}$ convection velocity. The significance of such a transformation is shown in Figure 1, where corotation is viewed from both the geographic inertial and the geomagnetic inertial frame. In both frames the locations of the geographic and geomagnetic poles are denoted by $g$ and $m$, respectively, the concentric circles represent lines of constant latitude about the central pole, and the solar direction is at 12 hours local time. The arrows $\mathrm{A}, \mathrm{B}$, and $\mathrm{C}$ represent corotation velocities of three different plasma elements along the dawn-dusk meridian. When these corotation velocities are transformed to the geomagnetic frame, not only does the speed change, but for vector $B$ there is an apparent reversal in flow direction. In fact, for all

PLASMA COROTATION VIEWED IN TWO DIFFERENT FRAMES

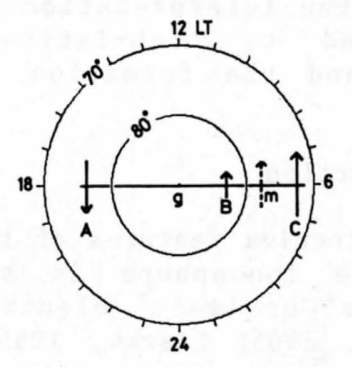

(a)

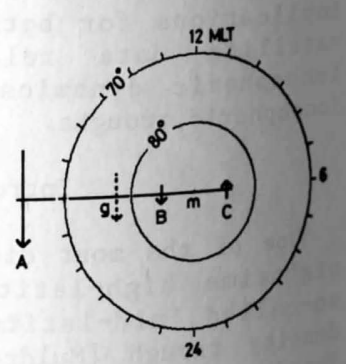

(b)
Fig. 1. Plasma corotation viewed in (a) geographic and (b) geomagnetic inertial frames. The arrows $\mathrm{A}, \mathrm{B}$, and $\mathrm{C}$ represent the plasma velocities at three points. The symbols $g$ and $m$ represent the geographic and geomagnetic poles, respectively. The dashe arrows represent the velocity vectors poles. 
plasma elements between the poles a sunward plasm in the geographic frame would appear to sot be antent shown in Figure 1. However, the most alignment fact to emerge from a careful study of iaportant is that plasma corotation about the pigure pole also appears as plasma geogration about the geomagnetic pole when iewed from the geomagnetic inertial frame. thus in this frame, corotation is UT independent.

View From the Geomagnetic Inertial Frame

In the geomagnetic inertial frame the mined action of plasma corotation and agnetospheric convection results in a flow attern similar to that shown in Figure $2 \mathrm{~b}$. In his geomagnetic frame the plasma streamlines coincide with the electric equipotentials, and the flow pattern is shown as a contour plot of equipotentials. The contours are drawn at 10-kilovolt $(\mathrm{kV})$ intervals in an inertial frame with the sun at 12 hours MLT. A total cross-tail potential drop of $64 \mathrm{kV}$ has been used in the model for the magnetospheric electric field. Lines of constant magnetic latitude are represented by concentric dashed circles, while agnetic longitude is represented by tick marks at hourly MLT intervals.

If the magnetospheric cross-tail potential is time independent, the convection pattern shown in Figure 2b will not vary with universal time, and at any instant the flow direction and speed distribution are as shown in Figure 2a. In this and subsequent figures the arrows are the same length and are used only to indicate flow direction. The speed distribution is shown by the gray scale display, in which the darkest tone represents the lowest horizontal speeds. In this geomagnetic frame the plasma flows in a two-cell pattern with antisunward flow over the polar cap and sunward flow at lower latitudes. The competition between eastward corotation and westward convection produces a stagnation point at 1800 MLT. Associated with the stagnation point is a band of low-speed flow, which lies symmetrically about 1800 MLT.

An important aspect of the convection pattern shown in Figure 2 concerns the time it takes a field tube of plasma to complete a full circulation. This circulation period will exhibit significant variation from trajectory to trajectory because the various trajectories have different lengths and the flow speed varies from one to the other. To illustrate this point, we show in Figure 3 the circulation times for eight representative plasma drift trajectories. The circulation times vary from a few hours for the smaller trajectories 3,7 , and 8 to much more than a day for trajectory 4, which passes through the band of low-speed flow. The lower-latitude trajectories 1 and 2 have a circulation period of 1 day, which indicates corotation about the geomagnetic pole in this geomagnetic inertial frame.

As was noted earlier, the plasma drift pattern shown in Figures 2 and 3 for the geomagnetic inertial frame does not vary with universal time, and therefore the circulation periods should be UT independent. To check this point, we calculated circulation times starting at different universal times and different positions, and indeed, the circulation periods are UT independent.
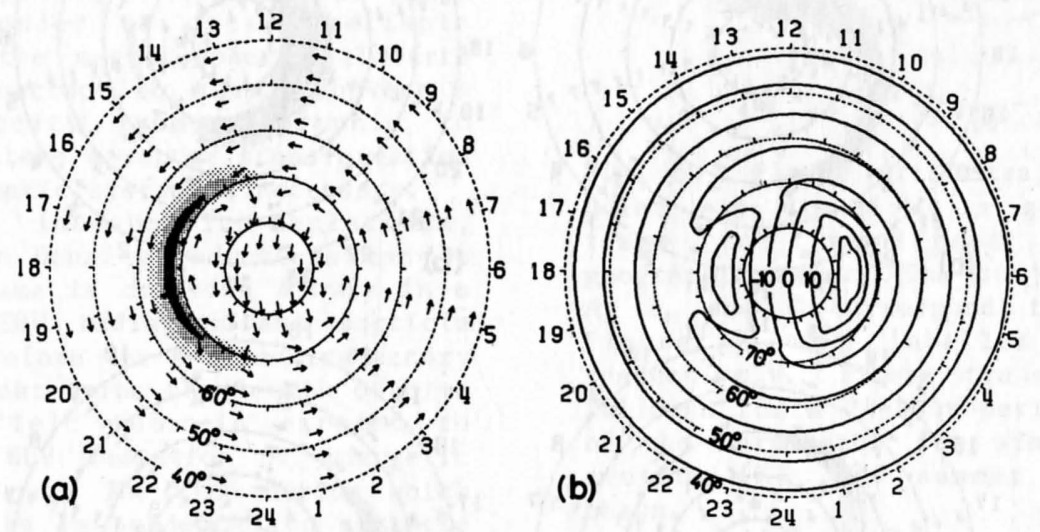

GRAY SCALE KEY

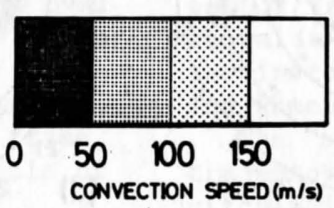

Fig. 2. (a) Plasma flow directions and speed distribution and (b) contours of electric potential over the polar cap viewed in a magnetic inertial frame. The total cross-tail potential is $64 \mathrm{kV}$. Magnetic longitude is represented by magnetic local time with the sun located at 1200 MLT. The dashed circles represent magnetic latitude. In Figure $2 a$ the shading corresponds to intervals of convection speed, the darkest tone corresponding to the lowest-speed interval, and the arrows are unit vectors indicating flow direction. In Figure $2 \mathrm{~b}$ the contour interval is $10 \mathrm{kV}$. 


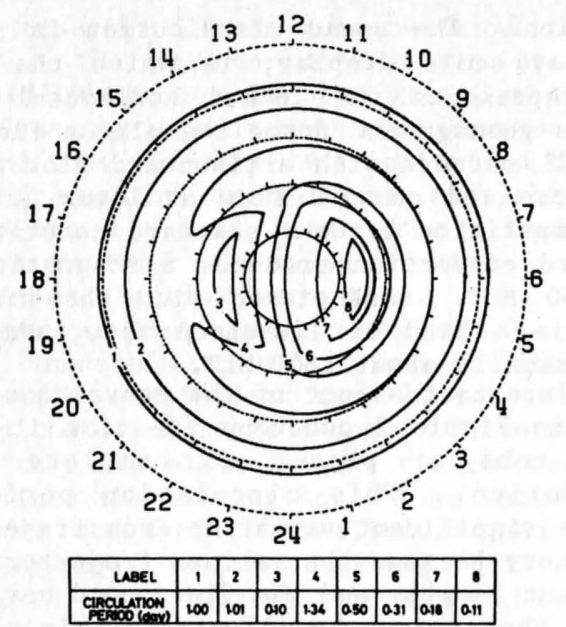

Fig. 3. Plasma drift trajectories over the polar cap viewed in a magnetic inertial frame. Each of the eight trajectories shown has a different circulation period, tabulated in the figure. For these calculations the total cross-tail potential was maintained at $64 \mathrm{kV}$.

\section{View From the Geographic Inertial Frame}

If the displacement between the geographic and geomagnetic poles is ignored, the plasma flow pattern predicted for the geographic inertial frame would be UT independent and identical to that shown in Figures 2 and However, the displacement between the and 3 . cannot be neglected, and the motion poles geomagnetic pole about the geographic pol the to introduce a UT dependence when the acts drift velocities are transformed frasma geomagnetic to the geographic frame.

This UT dependence is clearly illustrated in Figure 4, where we present flow directions in speed distributions in the geographic inertial frame for the four universal times correspial to the geomagnetic pole's being on thending midnight, (b) dawn, (c) noon, and (d) (a) meridians. In these and subsequent (d) dusk inertial plots the arrows and gray geographic defined in the same way as in Figure 2. Also, our definition of inertial frames is such tha, the sun is fixed at 12 hours in the geomagnetic frame but moves by approximately 1 degree per day in the geographic frame. This motion is sufficiently small that for our purposes the sun can be taken to be at 12 hours local time in both frames.

In addition to the obvious UT dependence shown in Figure 4, several other features are noteworthy. First, the band of low-speed plasma flow that is located symmetrically about 1800 MLT in the geomagnetic frame (Figure 2) is in general not symmetrical about $1800 \mathrm{LT}$ when viewed from the geographic frame. Furthermore, the region of very low speed flow ( $<50 \mathrm{~m} / \mathrm{s})$ moves from noon to midnight local time during the course of a day. Certain flow features are
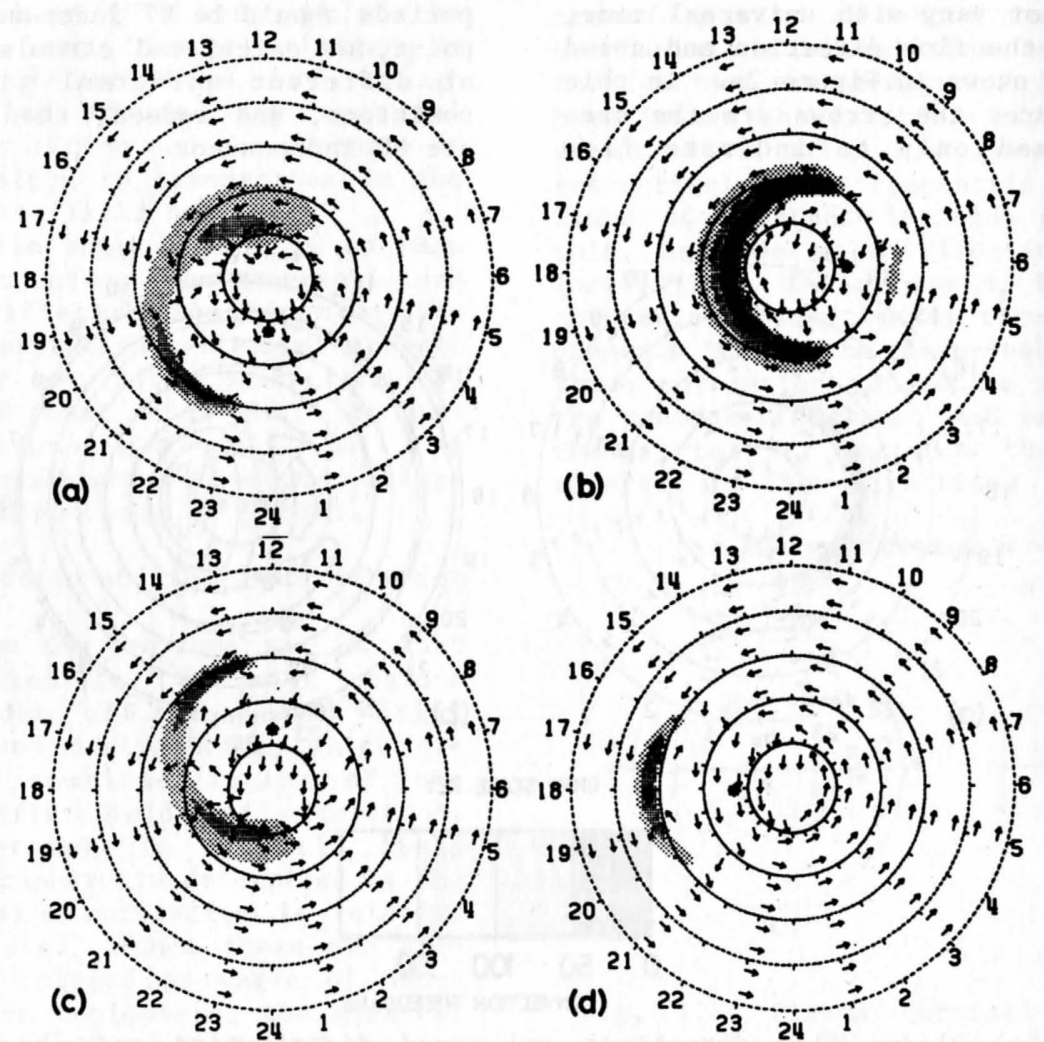

Fig. 4. Plasma flow directions and speed distributions viewed in the geographic inertial frame for nonaligned geomagnetic and geographic axes are shown for four universal times. The four universal times correspond to the location of the magnetic pole (shown by an asterisk) on the (a) midnight, (b) dawn, (c) noon, and (d) dusk meridians. The arrows and gray scaling are the same as in Figure 2 . The total cross-tail potential is $64 \mathrm{kV}$. 
also evident in the geographic frame at certain also ersal times, such as shear reversals, untational reversals, and throats. The hroatlike feature appears when the magnetic thre is on the midnight meridian (Figure 4a) and is located on the sunward side of the magnetic Rotational reversals of the plasma flow polection can be seen in the low-speed flow regions, and a high-speed shear reversal is evident at $80^{\circ}$ latitude on the noon meridian in pigure $4 \mathrm{~b}$. Also, the anti-sunward flow over the polar cap is not always parallel to the noon-midnight meridian in the geographic inertial frame.

It should be emphasized that the movement of the low-speed region with UT and the appearance f a throatlike feature at a certain UT are a consequence of the relative motion of the geomagnetic and geographic poles. These features are not evident in the geomagnetic inertial frame and hence are not connected with our model of the magnetospheric electric field.

The above results have very important implications for both satellite observations and ionospheric depletions. Considering first satellite observations, we note that high-inclination satellite orbits appear invariant on the time scale of a few days when viewed in the geographic inertial frame. A polar satellite orbit would produce the same track on all four panels of Figure 4. Consequently, the plasma flow, as observed by such a satellite, would appear to be strong1y UT dependent. Significant UT variations can occur during an orbital period ( $\sim 90 \mathrm{~min})$, and hence orbit to orbit variations can be expected even for quiescent magnetospheric conditions. Therefore the UT variation of the plasma drift pattern due to the relative motion of the geomagnetic and geographic poles must be taken into account in order to obtain reliable information about the magnetospheric electric field. It is not correct to simply convert a plasma drift velocity from geographic to geomagnetic coordinates; a vector transformation to the rotating magnetic system is necessary.

With regard to ionospheric depletions, significant electron density reductions can be expected if the plasma is drifting slowly in a region devoid of EUV radiation and particle precipitation. Therefore for a given trajectory It is important to determine the length of time that the plasma field tube is exposed to production due to EUV radiation or energetic particle precipitation. The time during which the plasma field tube is subjected to particle precipitation can best be determined in the agnetic inertial frame because the auroral oval apping is relative to the magnetic pole. Hower, the length of time that the plasma fald tube is exposed to sunlight can best be ince ined in the geographic inertial frame, few the terminator is fixed on a time scale of few days.

ohapes though the plasma trajectories have simple ore in the magnetic inertial frame, they are becauslex in the geographic inertial frame about of the motion of the geomagnetic pole In Pige geographic pole. This is illustrated

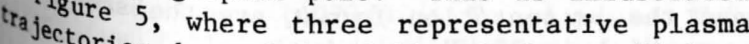
ctories have been followed for a 24-hour
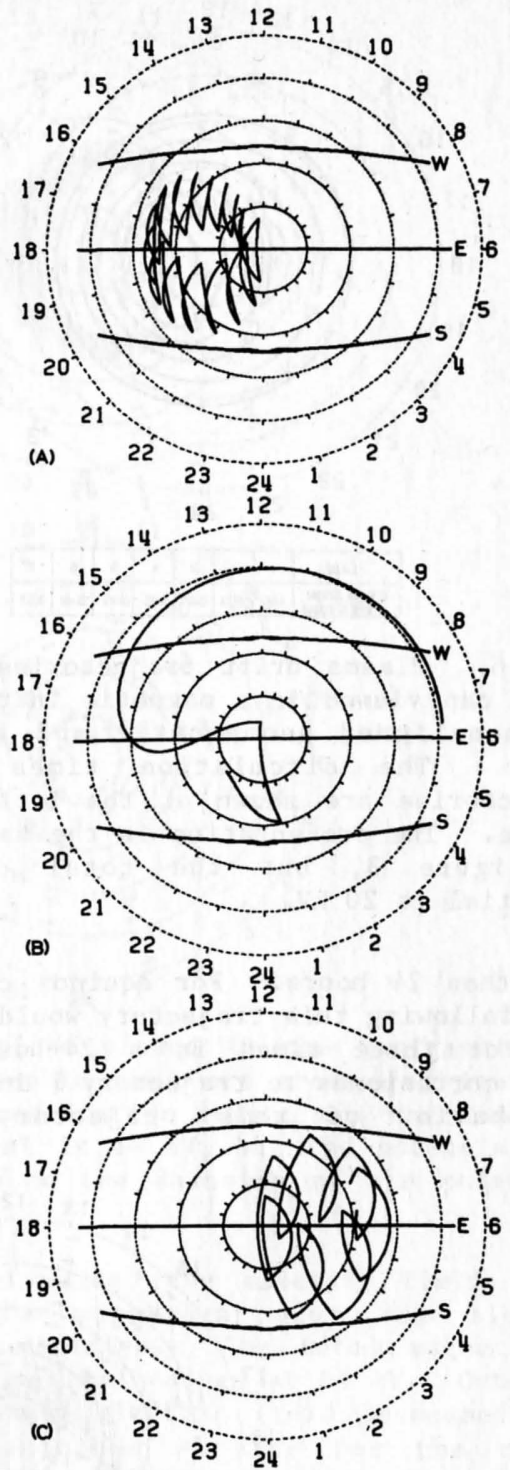

Fig. 5. Plasma drift trajectories over the polar cap viewed in a geographic inertial frame for nonaligned geomagnetic and geographic axes. The trajectories in panels $A, B$, and C correspond to those shown in Figure 3 and labelled 3, 4, and 7, respectively. These trajectories have been followed for a 24-hour period. The positions of the terminator for winter solstice (W), equinox (E), and summer solstice (S) are shown.

period in the geographic inertial frame. Also shown in this figure are the positions of the terminator at winter solstice (W), equinox (E), and summer solstice (S).

The trajectory in panel A corresponds to trajectory 3 in Figure 3 . This trajectory has a circulation period of about 2 hours, and hence the plasma executes many cycles per day. Depending on the location of the terminator, the p.lasma may drift entirely in darkness, entirely in sunlight, or move in and out of sunlight many times during the course of the day. The trajectory in panel $B$ corresponds to trajectory 4 in Figure 3 , and its circulation period is 


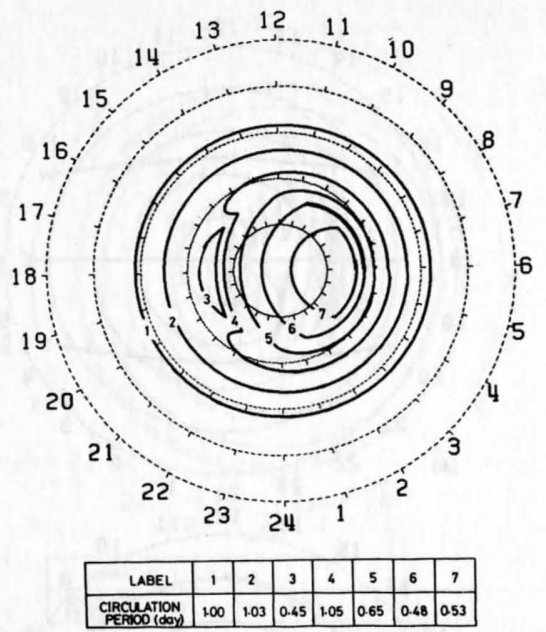

Fig. 6. Plasma drift trajectories over the polar cap viewed in a magnetic inertial frame for nonaligned geomagnetic and geographic axes. The circulation times for the trajectories are shown at the bottom of the figure. The presentation is the same as that in Figure 3, but the total cross-tail potential is $20 \mathrm{kV}$.

longer than 24 hours. For equinox conditions, plasma following this trajectory would cross the terminator three times in a 24-hour period. Panel C corresponds to trajectory 7 in Figure 3. The behavior of this trajectory in the geographic inertial frame is similar to that of trajectory 3, but its circulation period of longer, and hence fewer cycles are executed in
24 hours.

In each of the three panels, only one trajectory has been followed and for only one starting position and time. The net effect of following many such 'start points' for one of trajectory cell as defined in Figure 3 would ben to produce a broad band of latitudes and local times in which the trajectories are confined Looking at panel A, where the cells have a short circulation period, one can almost infer the band of latitudes and local times in which these different start point paths would lie. In fact, it would be approximately a ring centered on the dusk geographic meridian at about $76^{\circ}$ latitude.

\section{Effect of Different Uniform Magnetospheric Electric Fields}

In the model calculations discussed above, we used a total cross-tail magnetospheric potential of $64 \mathrm{kV}$, which corresponds to moderately active periods. We also considered magnetospheric potentials of 120 and $20 \mathrm{kV}$ to simulate very active and very quiet magnetic periods, respectively. For the very active case the results were similar to those found for the 64-kV calculation. The UT dependence of the low-speed regions and the flow directions were again present, although to a lesser extent.

For low magnetic activity $(20 \mathrm{kV})$ some interesting new features emerge. Figure 6 shows
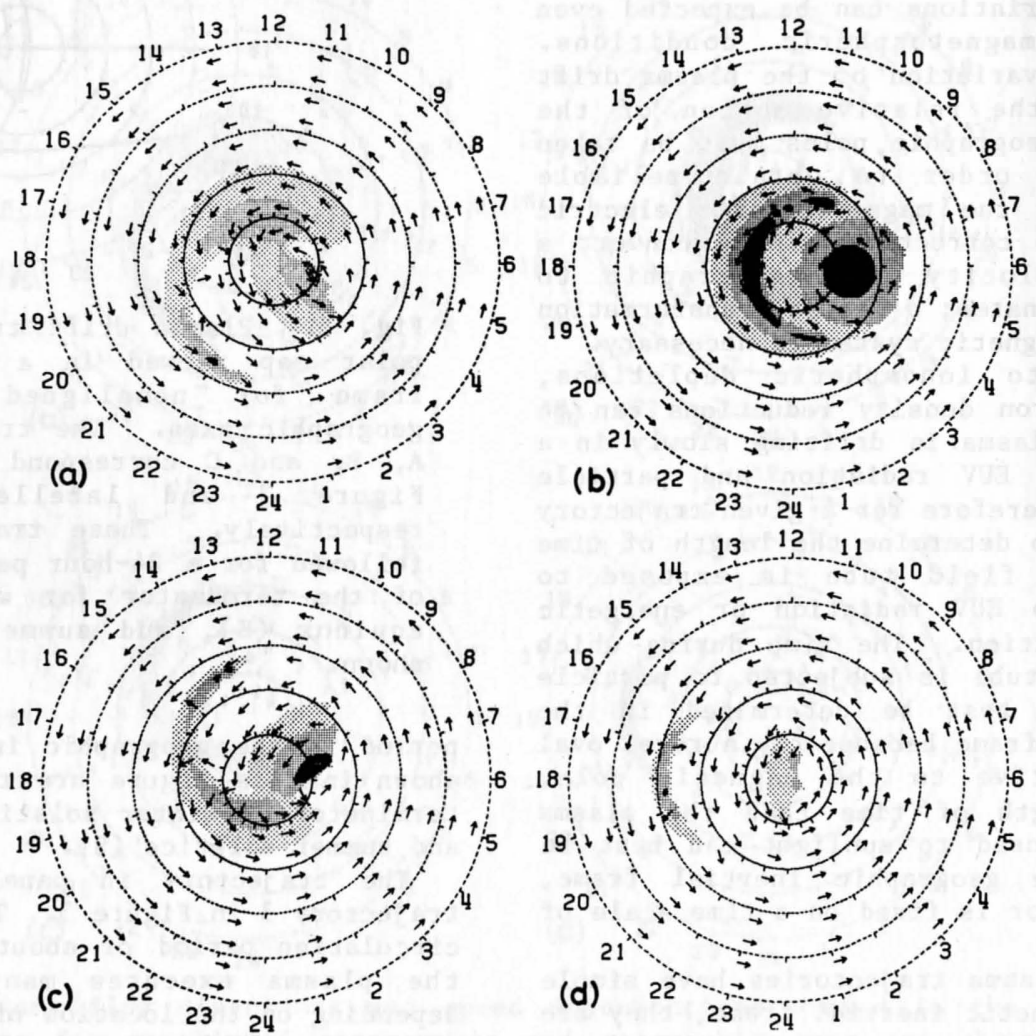

Fig. 7. Plasma flow directions and speed distributions viewed in the geographic inertial frame for nonaligned geomagnetic and geographic axes are shown for four universal times. The four universal times and the presentation format are the same as those in Figure 4. The total cross-tail potential is $20 \mathrm{kV}$. 

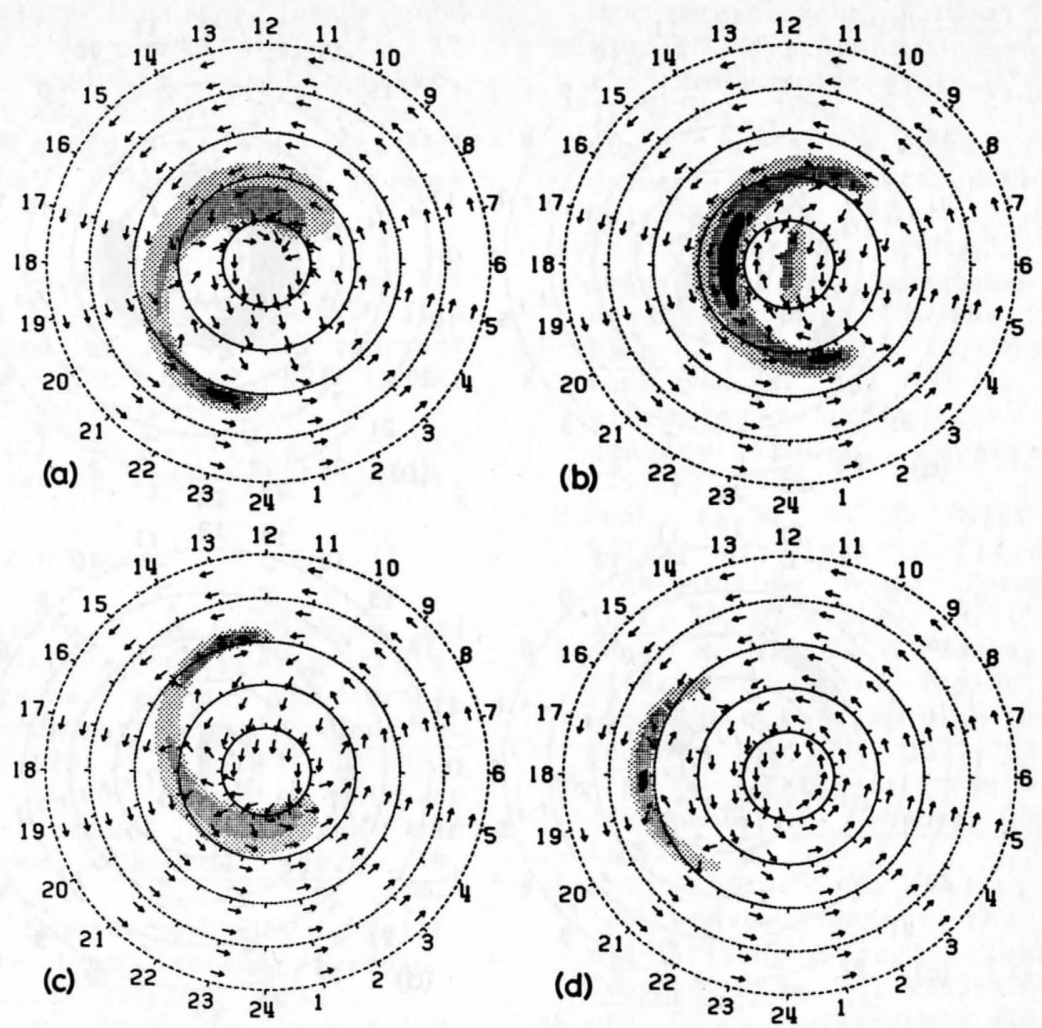

Fig. 8. Plasma flow directions and speed distributions viewed in the geographic inertial frame for nonaligned geomagnetic and geographic axes are shown for four universal times. The four universal times and the presentation format are the same as those in Figure 4. The total cross-tail potential is $64 \mathrm{kV}$, but the potential distribution is asymmetric with enhanced plasma flow on the dawnside of the polar cap.

the plasma drift trajectories in the magnetic inertial frame for a $20-\mathrm{kV}$ cross-tail potential. Also shown in this figure are the circulation periods for several representative trajectories. Unlike the $64-\mathrm{kV}$ case the plasma drift tends to be more like corotation, and the circulation times are much nearer the 1-day corotation period.

The view from the geographic inertial frame is shown in Figure 7 for the same four universal times that were used previously. The most evident new feature is the appearance of a large area of low-speed flow on the dawnside of the polar cap at certain universal times. This is ast noticeable when the magnetic pole lies near the dawn meridian (panel b). Another noteworthy feature is that the direction of plasma flow across the polar cap no longer lies close to the noon-midnight meridian as it did for the $64-\mathrm{kV}$ case but deviates by large angles from this aeridian (compare Figures 4 and 7 ).

\section{Effect of Asymmetric Magnetospheric}

The magnetospheric electric field is not al ways uniform across the polar cap but can be deped on either the dawnside or the duskside, compong on the direction of the azimutha? (see, of the interplanetary magnetic field [1977] for example, Fairfield [1977], Heppner effect, and Stern [1977]). To simulate this fect, we followed the suggestion of Heppner
[1977] and varied the electric field linearly across the magnetic polar cap along the dawn-dusk meridian. The total magnetospheric potential was maintained at $64 \mathrm{kV}$. Outside the polar cap the electric field decreased in the manner described earlier for the constant magnetospheric electric field.

As was the case with the previous electric field models, the plasma flow in the magnetic inertial frame was found to be invariant, the only important difference being enhanced flow on the dawnside or duskside of the polar cap. The plasma flow directions and speed distributions, as seen in the geographic inertial frame, are shown in Figures 8 and 9 for electric field enhancements on the dawnside and duskside of the polar cap, respectively. The plasma flow patterns shown in these figures are basically similar to those found for a uniform magnetospheric electric field, but there are a few interesting differences. First, the throatlike feature that appears in the postnoon sector of Figure $4 \mathrm{a}$ is in the prenoon sector in the case of an enhanced electric field on the dawnside (Figure 8a) and is absent in the case of an enhanced electric field on the duskside (Figure 9a). Also, for the dawn enhanced electric field case, low-speed regions can appear at postmidnight and prenoon times at latitudes outside the polar cap (Figure $8 \mathrm{~b}$ ). When the enhanced electric field is on the duskside, low-speed regions appear in the dawn polar regions (Figure 9). 

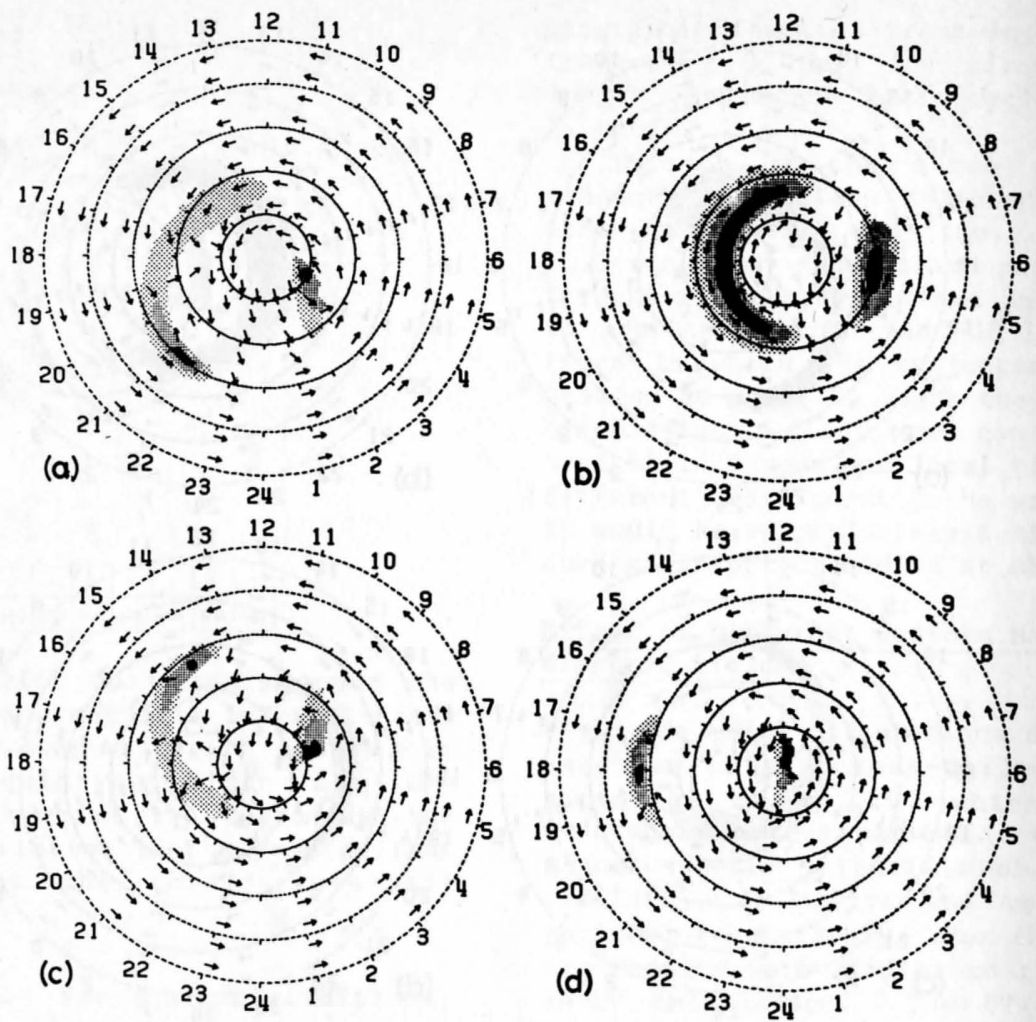

Fig. 9. Plasma flow directions and speed distributions viewed in the geographic inertial frame for nonaligned geomagnetic and geographic axes are shown for four universal times. The four universal times and the presentation format are the same as those in Figure 4. The total cross-tail potential is $64 \mathrm{kV}$, but the potential distribution is asymmetric with enhanced plasma flow on the duskside of the polar cap.

Summary

We have studied the effect that the displacement between the geomagnetic and geographic poles has on high-latitude plasma convection. From our study we have found the following:

1. Corotation of plasma about the geographic pole appears as corotation of plasma about the geomagnetic pole when viewed from a magnetic inertial reference frame.

2. In the magnetic inertial frame the combination of UT-independent corotation and a time-independent convection electric field produces a flow pattern that does not vary with universal time.

3. This UT-independent flow pattern becomes UT dependent when viewed in a geographic inertial frame because of the motion of the magnetic pole about the geographic pole.

4. The UT variation of the plasma flow pattern in the geographic inertial frame occurs on a time scale that is comparable to satellite orbital periods and that is much less than typical plasma flow times over the polar cap.

5. In the geographic inertial frame the main region of very low speed flow is not centered at. 1800 LT but moves from about 1300 to 2300 LT during the course of a day. The size and shape of this region also vary with universal time.

6. In the geographic inertial frame, additional low-speed regions occur in the morning sector at certain universal times.
7. In the geographic inertial frame a throatlike feature appears at certain universal times near noon and near midnight. This feature is seen only in the geographic inertial frame and is not connected with our model of the magnetospheric electric field. It is a consequence of the relative motion between the geographic and geomagnetic poles.

8. Low cross-tail electric potentials, which correspond to quiet geomagnetic activity, produce a plasma drift pattern which has a pronounced low-speed region in the polar cap in addition to the one that normally occurs between 1300 and $2.300 \mathrm{LT}$.

9. Large, uniform magnetospheric electric fields and asymmetric electric fields do not produce any significant new results beyond those listed above.

The above results were obtained for the northern hemisphere, where the angle between the geomagnetic and geographic axes is about 12 . Even larger effects can be anticipated for the southern hemisphere, where this angle is muct greater.

Finally, our results have important implications for both the interpretation of satellite data and the formation of plasu troughs. Since the plasma flow pattern is dependent in the geographic inertial frame owin to the relative motion of the geographic aken geomagnetic poles, this effect must be table into account in order to obtain rellabtc information about the magnetospheric elect 
field. Likewise, since the size, shape, and field. of the main low-speed region is UT location in the geographic inertial frame, dependealculations of the behavior of the 'main' oodel 'mid-latitude' trough must take this UT or atian into account if they are to produce reliable predictions.

Acknowledgments. We thank B. J. Watkins for helpful discussion concerning his work. This research was supported by Air Force contract rSAF/ESD F19628-79-C-0025 and NSF grant AM78-10501 to Utah State University.

The Editor thanks R. G. Roble for his ssistance in evaluating this brief report.

\section{References}

Ahmed, M., R. C. Sagalyn, P. J. L. Wildman, and W. J. Burke, Topside ionospheric trough morphology: Occurrence frequency and diurnal, seasonal, and altitude variations, J. Geophys. Res., 84, 489-498, 1979.

Axford, W. I., and C. O. Hines, A unifying theory of high-latitude phenomena and geomagnetic storms, Can. J. Phys., 39, 1433-1464, 1961 .

Banks, P. M. R. W. Schunk, and W. J. Raitt, $\mathrm{NO}^{+}$and $\mathrm{O}^{+}$in the high latitude F-region, Geophys. Res. Lett., 1, 239-242, 1974.

Brinton, H. C., J. M. Grebowsky, and L. H. Brace, The high-latitude winter $F$ region at $300 \mathrm{~km}$ : Thermal plasma observations from AE-C, J. Geophys. Res., 83, 4767-4776, 1978.

Fairfield, D. H., Electric and magnetic fields in the high-latitude magnetosphere, Rev. Geophys. Space Phys., 15, 285-298, 1977.

Feinblum, D. A. and R. J. Horan, Hilion - A model of the high-latitude ionospheric F2-layer and statistics of regular ionospheric effects at Ft. Churchill, 1968, report Be11 Lab., Murray Hil1, N.J., 1973.

Grebowsky, J. M., Y. Tulunay and A. J. Chen, Temporal variations in the dawn and dusk midlatitude trough and plasmapause position, Planet. Space Sci., 22, 1089-1099, 1974.

Heppner, J. P., Empirical models of high-latitude electric fields, J. Geophys. Res., 82, 1115-1125, 1977.

nudsen, W. C., Magnetospheric convection and the high-latitude $F_{2}$ ionosphere, J. Geophys. Res., 79, 1046-1055, ${ }^{2} 1974$.

nudsen, W. C., P. M. Banks, J. D. Winningham, and D. M. Klumpar, Numerical model of the convecting $\mathrm{F}_{2}$ ionosphere at high latitudes, $\mathrm{J}$. Geophys. Res., 82, 4784, 1977.

iszka, L., Variation according to latitude of the electron content of the ionosphere near the aurora1 zone, Nature, 208, 280-281, 1965.

Miller, N. J., The main electron trough during the rising solar cycle, J. Geophys. Res., 75, 7175-7181, 1970.

Muldrew, D. B., F layer ionization troughs deduced from Alouette data, J. Geophys. Res., 70, 2635-2650, 1965.

Rycroft, M. J, and J. 0. Thomas, The magnetospheric plasmapause and the electron density trough at the Alouette-I orbit, Planet. Space Sci., 18, 65-80, 1970.

Schunk, R. W. and P. M. Banks, Auroral $\mathrm{N}_{2}$ vibrational excitation and the electron density trough, Geophys. Res. Lett., 2 , 239-242, 1975.

Schunk, R. W., W. J. Raitt and P. M. Banks, Effect of electric fields on the daytime high-latitude E-and F-regions, J. Geophys. Res., 80, 3121-3130, 1975.

Schunk, R. W., P. M. Banks, and W. J. Raitt, Effects of electric fields and other processes upon the nighttime high-latitude $\mathrm{F}$ layer, J. Geophys. Res., 81, 3271-3282, 1976.

Sharp, G. W., Mid-1atitude trough in the night ionosphere, J. Geophys. Res., 71, 1345-1356, 1966.

Spiro, R. W., R. A. Heelis, and W. B. Hanson, Ion convection and the formation of the mid-latitude $\mathrm{F}$ region ionization trough, J. Geophys. Res., 83, 4255-4264, 1978.

Stern, D. P., Large-scale electric fields in the earth's magnetosphere, Rev. Geophys. Space Phys., 15, 156-194, 1977.

Tulunay, Y., and J. Sayers, Characteristics of the mid-latitude trough as determined by the electron density experiment on Ariel III, J. Atmos. Terr. Phys., 33, 1737, 1971.

Volland, H., Models of global electric fields within the magnetosphere, Ann. Geophys., 31, $159,1975$.

Volland, H., A model of the magnetospheric electric convection field, J. Geophys. Res., 83, 2695-2699, 1978.

Wagner, R. A., A. L. Snyder, and S.-I. Akasofu, The structure of the polar ionosphere during exceptionally quiet periods, Planet. Space Sci, 21, 1911, 1973.

Watkins, B. J., A numerical computer investigation of the polar F-region ionosphere, Planet. Space Sci., 26, 559-569, 1978.

Wrenn, G. L. and W. J. Raitt, In situ observations of mid-latitude ionospheric phenomena associated with the plasmapause, Ann. Geophys., 31, 17-28, 1975.

(Received April 18, 1979, accepted May 24, 1979) 